\title{
A Micromegas Detector for Neutron Beam Imaging at the n_TOF Facility at CERN
}

F. Belloni,${ }^{1, *}$ S. Andriamonje, ${ }^{2}$ E. Berthoumieux,${ }^{1}$ M. Calviani,${ }^{2}$ E. Chiaveri,${ }^{2}$ N. Colonna,${ }^{3}$ Y. Giomataris ${ }^{1}$ C. Guerrero, ${ }^{2}$ F. Gunsing, ${ }^{1}$ F.J. Iguaz, ${ }^{1}$ M. Kebbiri, ${ }^{1}$ J. Pancin, ${ }^{1}$ T. Papaevangelou,,${ }^{1}$ A. Tsinganis,${ }^{2,4}$ V. Vlachoudis, ${ }^{2}$ S. Altstadt,${ }^{5}$ J. Andrzejewski,${ }^{6}$ L. Audouin, ${ }^{7}$ M. Barbagallo,${ }^{3}$ V. Bécares,${ }^{8}$ F. Bečváŕ,${ }^{9}$ J. Billowes, ${ }^{10}$ V. Boccone, ${ }^{2}$ D. Bosnar, ${ }^{11}$ M. Brugger, ${ }^{2}$ F. Calviño, ${ }^{12}$ D. Cano-Ott, ${ }^{8}$ C. Carrapiço, ${ }^{13}$ F. Cerutti, ${ }^{2}$ E. Chiaveri, ${ }^{1,2}$ M. Chin, ${ }^{2}$ G. Cortés, ${ }^{12}$ M.A. Corté-Giraldo, ${ }^{14}$ M. Diakaki,${ }^{4}$ C. Domingo-Pardo, ${ }^{15}$ I. Duran,${ }^{16}$ N. Dzysiuk, ${ }^{17}$ C. Eleftheriadis, ${ }^{18}$ A. Ferrari, ${ }^{2}$ K. Fraval,${ }^{1}$ S. Ganesan,${ }^{19}$ A.R. García,${ }^{8}$ G. Giubrone,${ }^{15}$ M.B. Gómez-Hornillos, ${ }^{12}$ I.F. Gonçalves,${ }^{13}$ E. González-Romero, ${ }^{8}$ E. Griesmayer,${ }^{20}$ P. Gurusamy,${ }^{19}$ D.G. Jenkins, ${ }^{21}$ E. Jericha, ${ }^{20}$ Y. Kadi, ${ }^{2}$ F. Käppeler ${ }^{22}$ D. Karadimos ${ }^{4}{ }^{4}$ P. Koehler, ${ }^{23}$ M. Kokkoris ${ }^{4}$ M. Krtička, ${ }^{9}$ J. Kroll, ${ }^{9}$ C. Langer, ${ }^{5}$ C. Lederer,${ }^{5,24}$ H. Leeb, ${ }^{20}$ L.S. Leong, ${ }^{7}$ R. Losito, ${ }^{2}$ A. Manousos,${ }^{18}$ J. Marganiec, ${ }^{6}$ T. Marítnez, ${ }^{8}$ C. Massimi, ${ }^{25}$ P.F. Mastinu,${ }^{17}$ M. Mastromarco,${ }^{3}$ M. Meaze,${ }^{3}$ E. Mendoza, ${ }^{8}$ A. Mengoni, ${ }^{26}$ P.M. Milazzo,${ }^{27}$ F. Mingrone,${ }^{25}$ M. Mirea,${ }^{28}$ W. Mondalaers,${ }^{29}$ C. Paradela,,${ }^{16}$ A. Pavlik,${ }^{24}$ J. Perkowski, ${ }^{6}$ A.J.M. Plompen ${ }^{29}$ J. Praena,${ }^{14}$ J.M. Quesada, ${ }^{14}$ T. Rauscher,${ }^{30}$ R. Reifarth,${ }^{5}$ A. Riego, ${ }^{12}$ F. Roman, ${ }^{2,}{ }^{28}$ C. Rubbia, ${ }^{2,31}$ R. Sarmento, ${ }^{13}$ P. Schillebeeckx,${ }^{29}$ S. Schmidt,${ }^{5}$ G. Tagliente,${ }^{3}$ J.L. Tain,${ }^{15}$ D. Tarrío, ${ }^{16}$ L. Tassan-Got, ${ }^{7}$ S. Valenta, ${ }^{9}$ G. Vannini,,${ }^{25}$ V. Variale,${ }^{3}$ P. Vaz,${ }^{13}$ A. Ventura, ${ }^{26}$ R. Versaci, ${ }^{2}$ M.J. Vermeulen, ${ }^{21}$ R. Vlastou, ${ }^{4}$ A. Wallner,${ }^{24}$ T. Ware,${ }^{10}$ M. Weigand,${ }^{5}$ C. Weiss,${ }^{20}$ T.J. Wright,${ }^{10}$ and P. Žugec ${ }^{11}$

${ }^{1}$ CEA - Saclay, Irfu, F-91191 Gif-sur-Yvette, France

${ }^{2}$ European Organization for Nuclear Research (CERN), Geneva, Switzerland

${ }^{3}$ Istituto Nazionale di Fisica Nucleare, Bari, Italy

${ }^{4}$ National Technical University of Athens (NTUA), Athens, Greece

${ }^{5}$ Johann-Wolfgang-Goethe Universität, Frankfurt, Germany

${ }^{6}$ Uniwersyte Eódzki, Lodz, Poland

${ }^{7}$ Centre National de la Recherche Scientifique/IN2P3 - IPN, Orsay, France

${ }^{8}$ Centro de Investigaciones Energeticas Medioambientales y Tecnólgicas (CIEMAT), Madrid, Spain

${ }^{9}$ Charles University, Prague, Czech Republic

${ }^{10}$ University of Manchester, Oxford Road, Manchester, UK

${ }^{11}$ Department of Physics, Faculty of Science, University of Zagreb, Croatia

${ }^{12}$ Universitat Politecnica de Catalunya, Barcelona, Spain

${ }^{13}$ Instituto Tecnológico e Nuclear, Instituto Superior Técnico, Universidade Técnica de Lisboa, Lisboa, Portugal

${ }^{14}$ Universidad de Sevilla, Spain

${ }^{15}$ Instituto de Fisica Corpuscular, CSIC-Universidad de Valencia, Spain

${ }^{16}$ Universidade de Santiago de Compostela, Spain

${ }^{17}$ Istituto Nazionale di Fisica Nucleare, Laboratori Nazionali di Legnaro, Italy

${ }^{18}$ Aristotle University of Thessaloniki, Thessaloniki, Greece

${ }^{19}$ Bhabha Atomic Research Centre (BARC), Mumbai, India

${ }^{20}$ Atominstitut, Technische Universität Wien, Austria

${ }^{21}$ University of York, Heslington, York, UK

${ }^{22}$ Karlsruhe Institute of Technology, Campus Nord, Institut für Kernphysik, Karlsruhe, Germany

${ }^{23}$ Oak Ridge National Laboratory (ORNL), Oak Ridge, TN 37831, USA

${ }^{24}$ University of Vienna, Faculty of Physics, Austria

${ }^{25}$ Dipartimento di Fisica, Universitá di Bologna, and Sezione INFN di Bologna, Italy

${ }^{26}$ Agenzia nazionale per le nuove tecnologie, l'energia e lo sviluppo economico sostenibile (ENEA), Bologna, Italy

${ }^{27}$ Istituto Nazionale di Fisica Nucleare, Trieste, Italy

${ }^{28}$ Horia Hulubei National Institute of Physics and Nuclear Engineering - IFIN HH, Bucharest - Magurele, Romania

${ }^{29}$ European Commission JRC, Institute for Reference Materials and Measurements, Retieseweg 111, B-2440 Geel, Belgium

${ }^{30}$ University of Basel, Basel, Switzerland

${ }^{31}$ Laboratori Nazionali del Gran Sasso dell'INFN, Assergi (AQ), Italy 
of one ionization and one proportional chamber. A micromesh separates the two communicating regions, where two different electric fields establish respectively a charge drift and a charge multiplication regime. The $\mathrm{n}_{-} \mathrm{TOF}$ facility at CERN provides a white neutron beam (from thermal up to $\mathrm{GeV}$ neutrons) for neutron induced cross section measurements. These measurements need a perfect knowlodge of the incident neutron beam, in particular regarding its spatial profile. A position sensitive micromegas detector equipped with a ${ }^{10} \mathrm{~B}$ based neutron/charged particle converter has been extensively used at the $\mathrm{n}_{-} \mathrm{TOF}$ facility for characterizing the neutron beam profile and extracting the beam interception factor for samples of different size. The boron converter allowed to scan the energy region of interest for neutron induced capture reactions as a function of the neutron energy, determined by the time of flight. Experimental results will be presented and compared to simulations, performed by means of the FLUKA code.

\section{INTRODUCTION}

The $\mathrm{n}_{-}$TOF facility [1] at CERN is a high fluence spallation neutron source aiming at $(n, f),(n, \gamma)$ and (n,charged particle) cross section measurements. The number of reactions induced in a target by an incident neutron beam is proportional to its intensity, which at n_TOF is accurately determined with two flux monitors. When the diameter of the incident neutron beam is wider than the lateral dimensions of the sample, only a fraction of all incident neutrons intercepts it and can induce a reaction. In this case, an effective neutron flux $\Phi_{\text {eff }}\left(E_{n}\right)=\Phi_{g l o b}\left(\mathrm{E}_{n}\right) \int_{A} \mathrm{k}\left(\mathrm{x}, \mathrm{y}, \mathrm{E}_{\mathrm{n}}\right) \mathrm{d} A$ has to be determined, with $\int_{A} \mathrm{k}\left(\mathrm{x}, \mathrm{y}, \mathrm{E}_{\mathrm{n}}\right) \mathrm{d} A / \int_{\infty} \mathrm{k}\left(\mathrm{x}, \mathrm{y}, \mathrm{E}_{\mathrm{n}}\right) \mathrm{d} A=$ b.i.f. $\left(\mathrm{E}_{n}\right)$, where $\Phi_{\text {glob }}$ represents the global neutron flux, $k\left(x, y, E_{n}\right)$ is the function describing the shape of the beam profile, and b.i.f. $\left(\mathrm{E}_{n}\right)$ is the beam interception factor, defined as the ratio of the number of neutrons intercepting the target to the number of neutrons in the beam.

The value of the beam interception factor is a function of the geometry of the beam and of the target and it is therefore measured with a position sensitive detector. The study of the shape of the beam profile is moreover a tool for checking possible beam misalignments.

At $\mathrm{n}_{-} \mathrm{TOF}$ a micromegas detector has been chosen for performing beam imaging and measuring the beam interception factor.

\section{EXPERIMENTAL SETUP}

Micromegas detectors [2] are gas detectors consisting of a stack of one ionization chamber and one proportional chamber separated by a subtle mesh. When a charged particle passes through the gas, electron and ion pairs are created and drift towards electrodes in opposite directions under the effect of an electric field. The electrons reach the mesh and pass through entering the proportional region where they are multiplied. The signal can be extracted both from the anode and from the mesh. On the basis of the building procedure, we distinguish among

\footnotetext{
* Corresponding author: francesca.belloni@cea.fr
}

simple micromegas detectors, bulk [3] type micromegas and microbulk [4] type micromegas.

The first n_TOF beam profiler was a micromegas detector of bulk type, with a radius of $4 \mathrm{~cm}$ and a square anode of $6 \mathrm{~cm}$ side segmented in 2 orthogonal directions by 106 strips. The detector was equipped with a neutron to charged particle converter $2 \mu \mathrm{m}$ thick in the form of $\mathrm{BC}_{4}$ enriched in ${ }^{10} \mathrm{~B}$ and it was filled with $\mathrm{Ar}(88 \%)$ $\mathrm{CF}_{4}(10 \%)$ and $\mathrm{C}_{4} \mathrm{H}_{10}$. Gassiplex cards performed the readout of the anode.

The second $\mathrm{n}_{-} \mathrm{TOF}$ beam profiler is a pixelized micromegas detector of $5 \mathrm{~cm}$ diameter. Each quarter of the detector is composed by 77 pixels with a pitch of 2.5 $\mathrm{mm}$. The detector was operated with the same converter, gas and electronics as the previous one.

\section{ANALYSIS}

Whenever a neutron interacts with the converter, an $\alpha$ particle and a ${ }^{7} \mathrm{Li}$ nucleus are produced. One of these two particles (or sometimes both) enter the gas and induce a signal both in the mesh and in the anode. The mesh signal is used to trigger the readout of the anode and gives the time when a neutron interacted with the converter. This time is translated into kinetic energy of the neutron using the time of flight technique. The mesh (black line) and anode (red line) signals, as read by the gassiplex, corresponding to a $7 \mathrm{Li}$ nuclei are displayed in Fig 1.

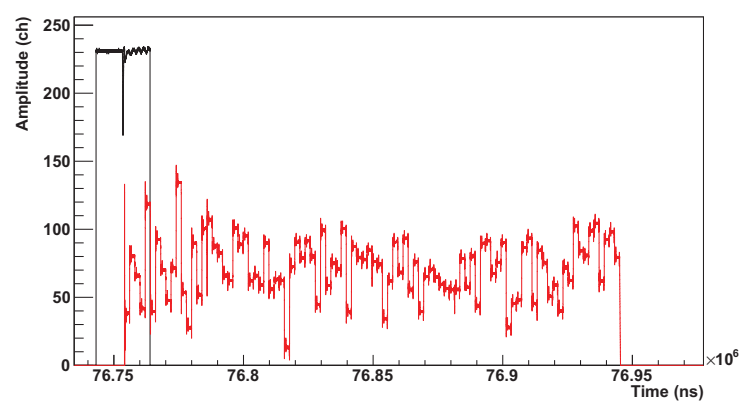

FIG. 1. Signal generated by a ${ }^{7} \mathrm{Li}$ particle in the mesh (black line) and in the anode (red line) as read by one gassiplex card.

In order to determine the point of interaction between 
the incident neutron and the converter, the signal aquired by the gassiplex has to be treated. A pedestal run (detector fed by high voltage but no neutron beam present) is performed, allowing to determine the baseline level in each strip (or pixel). This baseline is subsequently subtracted from the signal aquired in presence of the beam, strip by strip (or pixel by pixel). A threshold is applied to the resulting signal and all the strips (or pixels) showing a residual higher than such threshold are taken into account. A routine for cluster recognition is subsequently applied, to discard strips (or pixels) too far away from each other and clusters of too high multiplicity. The interaction point is in the end obtained by calculating the center of charge of the clusters.

\section{RESULTS}

\section{A. Strip Detector}

The beam profile extracted from the strip detector was not at all symmetric. This was partly imputed to a misalignment of the $\mathrm{n}_{-} \mathrm{TOF}$ beam optics and partly to a defect of construction of the detector. It was therefore decided not to employ the strip detector to extract the beam profile, but just as a flux monitor: the signal was not extracted from the strips, but only from the mesh and the number of interactions between the neutron beam and the converter was monitored for different movements of the movable collimator of the $n_{-}$TOF facility. The alignment position, corresponding to the maximization of the number of interactions, was found in correspondence of a tilt upstream of the collimator of $-8 \mathrm{~mm}$ and and $-6 \mathrm{~mm}$ downstream. After aligning the n_TOF beam optics, the strip detector was replaced by a new beam profiler with a pixelized readout.

\section{B. Pixelized Detector}

The shape of the beam profile extracted from the pixelized micromegas detector proved the achieved alignment of the beam optics.
The comparison between the beam interception factor experimentally determined and the one obtained by running FLUKA $[1,5]$ simulations is shown in Fig. 2 as a function of the incident neutron energy for 2 different target radii. The agreement between simulations and experimental data is within $4 \%$, but at low energies, where simulations do not take into account the neutrons scattered in the experimental area, and at high energies, where more statistics is needed.

\section{CONCLUSIONS}

Two micromegas detectors of bulk type have succefully been used for aligning the beam line, studying the beam

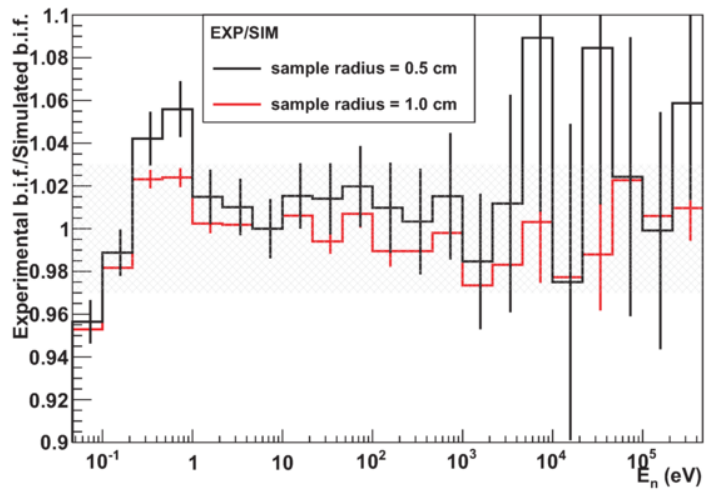

FIG. 2. Ratio of the experimentally determined beam interception factor to the simulated beam interception factor.

profile and extracting the beam interception factor at the $\mathrm{n}_{-}$TOF facility at CERN. The results of the the beam interception factor experimentally determined and of the one obtained by running FLUKA simulations show a good agreement. The discrepancies at high neutron energies can be reduced by increasing the statistics. At low neutron energies additional simulations are being performed. A pointwise agreement within 3\% is reached in the region of interest.
[1] C. Guerrero et al., Eur. Phys. J. A, 49 (2013).

[2] Y. Giomataris, Nucl. Instrum. Meth. A 419, 239 (1998).
[3] Y. Giomataris et al., Nucl. Instrum. Meth. A 560, 405 (2006).

[4] S. Andriamonje, J. of Instrum. 5, P02001 (2010).

[5] A. Fassò et al., Report numbers CERN-2005-10, 2005, INFN/TC_05/11, SLAC-R-773 (2005). 